\title{
Evaluación del ponticulus posticus según la relación esquelética en radiografías laterales estrictas
}

\section{Evaluation of ponticulus posticus according to the skeletal relationship found in strict lateral radiographs}

\author{
Jonathan Cook García Blásquez ${ }^{1, a, b}$, Alexis Evangelista-Alva ${ }^{2, a, c}$, Milushka Miroslava Quezada \\ Márquez ${ }^{2, a, b, c}$
}

\section{RESUMEN}

Objetivo: Evaluar el ponticulus posticus según la relación esquelética encontrada en radiografías laterales estrictas en la Centro Dental Docente de la Universidad Peruana Cayetano Heredia durante el período 2015 2017, utilizando la clasificación de acuerdo al grado de mineralización descrita por Selby y la clasificación de la relación esquelética de Steiner. Material y Métodos: Se realizó en radiografías laterales estrictas digitales usando una pantalla de 20 pulgadas utilizando el programa SIDEXIS XG, observando el grado de mineralización del ponticulus posticus: sin evidencia de la espícula ósea sobre la arteria vertebral = puente ausente, cuando se notaba o evidenciaba la formación de la espícula y/o calcificación en medio del puente o en forma incompleta = puente parcial, cuando el arco óseo estaba evidente terminado visualizándose = puente completo y la clasificación de la relación esquelética midiendo el ángulo ANB: Clase I $=0-4^{\circ}$; Clase II $=>4^{\circ}$ y Clase III $=\left\langle 0^{\circ}\right.$ : el análisis estadístico se hizo con el programa SPSS V.22.0 para Windows mediante las pruebas de Chi ${ }^{2}$. Resultados: De las 925 radiografías laterales estrictas digitales evaluadas se encontró que 283 radiografías presentaron ponticulus posticus y la mayor frecuencia se encontró en el tipo ausente $(69,4 \%)$, el tipo parcial $(17,1 \%)$ y el tipo completa (13.5\%). El ponticulus posticus se presentó en $25.1 \%$ del sexo femenino y en $38.4 \%$ en el sexo masculino. La relación esquelética asociada al ponticulus posticus se presentó en la Clase II (19.1\%), Clase I (10,4\%) y Clase III (1.1\%), Conclusiones: El ponticulus posticus es una variante anatómica que se presenta en el 30,6\% de los casos. No se encontró diferencia estadísticamente significativa entre la presencia de ponticulus posticus y la relación esquelética o sexo.

PALABRAS CLAVE: Radiografía, Ponticulus Posticus, Atlas. 


\section{SUMMARY}

Objective: To evaluate the posticus ponticulus according to the skeletal relationship found in strict lateral radiographs at the Teaching Dental Center of the Cayetano Heredia Peruvian University during the period 20152017, using the classification according to the degree of mineralization described by Selby and the classification of the Steiner's skeletal relationship. Material and Methods: It was performed on strict digital lateral radiographs using a 20-inch screen using the SIDEXIS XG program, observing the degree of mineralization of ponticulus posticus: no evidence of the bone spicule on the vertebral artery $=$ absent bridge, when it was noted or evidenced the formation of the spicule and / or calcification in the middle of the bridge or in incomplete form = partial bridge, when the bone arch was clearly finished visualizing = full bridge and the classification of the skeletal relationship by measuring the ANB angle: Class I $=0$ - 4th; Class II $=>4$ th and Class III $=\left\langle 0^{\circ}\right.$ : the statistical analysis was done with the SPSS V.22.0 program for Windows using the Chi2 tests. Results: Of the 925 digital strict lateral radiographs evaluated, it was found that 283 radiographs presented ponticulus posticus and the highest frequency was found in the absent type (69.4\%), the partial type (17.1\%) and the complete type (13.5\%). Ponticulus posticus occurred in $25.1 \%$ of the female sex and in $38.4 \%$ of the male sex. The skeletal relationship associated with ponticulus posticus was presented in Class II (19.1\%), Class I (10.4\%) and Class III (1.1\%). Conclusions: Ponticulus posticus is an anatomical variant that occurs in $30.6 \%$ of the cases. No statistically significant difference was found between the presence of ponticulus posticus and the skeletal relationship or sex.

KEYWORDS: Radiography, Ponticulus Posticus, Atlas.

\section{INTRODUCCIÓN}

Las radiografías laterales estrictas se utilizan en ortodoncia para evaluar el desarrollo, crecimiento y las relaciones morfométricas de las estructuras craneofaciales y dentales, también brindan información de diagnóstico sobre la región alta de la columna (1).

Las vértebras cervicales muestran gran variabilidad por ser más pequeñas y más delicadas que las verdaderas. La primera vértebra cervical (C1), difiere en estructura de todas las demás cervicales porque está desprovista del cuerpo y de la espina dorsal, según muchos autores es la más variable en el ser humano y en ella se encuentra un ligamento oblicuo que se calcifica parcial o completamente, es una osificación anómala, en forma de arco óseo que se extiende desde la masa lateral hasta el margen posteromedial de la ranura de la arteria vertebral (AV), que se puede ver en la radiografía simple del cráneo, en proyección lateral, sin embargo, generalmente la porción mastoidea del hueso temporal dificulta su buena observación (2).

El ponticulus posticus (PP) no ha tenido una atención adecuada en la anatomía radiográfica de la región de la columna cervical y su posible relación con algunas patologías. La importancia clínica potencial de la formación del PP es hasta la fecha controvertida; sin embargo, se le atribuye patologías tales como la migraña sin aura, cefaleas crónicas, vértigo, diplopía y dolor del cuello. Algunos autores lo asocian a la compresión de la $\mathrm{AV}$, insuficiencia vértebra-basilar o disección de la AV(3).

Por este motivo, el propósito de esta investigación fue evaluar el ponticulus posticus (PP) según la relación esquelética encontradas en radiografías laterales estrictas digitales (RLED) en el Centro Dental Docente, de la Universidad Peruana Cayetano Heredia, durante el periodo 2015-2017.

\section{MATERIAL Y MÉTODOS}

El diseño del presente estudio fue retrospectivo, transversal y descriptivo. La población fue 971 RLED del Servicio de Radiología Oral y Maxilofacial, de la sede de San Isidro, de la Universidad Peruana Cayetano Heredia durante los años 2015-2017.

La muestra fue elegida de la población por conveniencia (no probabilística), y fueron las que cumplían con los criterios de selección, quedando 925 RLED, el procedimiento fue: se incluyeron las radiografías nítidas de pacientes con edades entre 8 a 
80 años, de ambos sexos. Se excluyeron las RLED de pacientes con alteraciones morfológicas en columna cervical, las RLED de pacientes con patologías evidentes en el área a evaluar, las RLED de pacientes con superposición de la apófisis mastoides sobre el arco posterior del atlas $(\mathrm{C} 1)$.

La variable PP cuya definición conceptual y operacional es: Configuración morfológica ósea, descrita como variante anatómica que conecta el tubérculo retroglenoideo localizado en la parte posterior de la fosa articular superior del atlas con su arco posterior, que se origina por la osificación completa o incompleta de la membrana posterior atlanto-occipital sobre la ranura de la AV dando lugar a la formación de un hueco o forado que contiene la AV y la rama posterior del nervio espinal de la C1. La medición de esta variable se determinó mediante la evaluación de las RLED, considerando su escala de medición nominal con los siguientes valores: No presenta: cuando no se observa ninguna mineralización en el ligamento atlanto-occipital, total: cuando forma un anillo óseo completo del ligamento atlanto-occipital y la mineralización total que se extiende desde la masa lateral hasta el margen posteromedial de la ranura de la artería vertebral y parcial: cuando existe una mineralización lineal o amorfa del ligamento atlanto-occipital y la mineralización parcial que se extiende desde la masa lateral sin llegar al margen posteromedial de la ranura de la artería vertebral (figura 1).

La variable Relación Esquelética cuya definición conceptual y operacional: es la relación maxilar con la base del cráneo anteroposterior. La medición de esta variable se determinó mediante la evaluación de la RLED y la medición del ángulo ANB. Su escala de medición fue nominal a través de los siguientes valores: Clase I: cuando presenta una normorelación entre el maxilar y la mandíbula y se limita sólo a malposiciones dentarias. Generalmente asociado con perfilrectoolevementeconvexo. ClaseII:secaracteriza por presentar exceso esquelético maxilar o también llamado prognatismo maxilar, exceso dentoalveolar superior, deficiencia esquelética mandibular o también llamado retrognatismo mandibular y/o deficiencia dentoalveoiar inferior. Asociado con perfil facial convexo. Clase III: encontramos de igual manera las mismas desproporciones citadas en la clase II, pero con la dirección de desviación invertida; encontrando un retrognatismo maxilar y/o un prognatismo mandibular, caracterizado por una concavidad en el perfil facial. Además, se consideraron las variables edad y sexo, evaluadas a través de sus frecuencias.

Previamente se calibró al observador con un especialista en radiología oral y maxilofacial (Gold Estándar) en la identificación de los tipos del PP y medición del ángulo ANB en RLED. Para determinar que el observador fue calibrado, se realizó una serie de observaciones y se compararon con los criterios del calibrador hasta presentar un índice de Kappa y el coeficiente de correlación interclase (CCI) mayor a 0,80 . Se solicitó el permiso correspondiente al Departamento Académico de Medicina y Cirugía Bucomaxilofacial.

Para seleccionar las RLED se procedió a realizar una solicitud formal al Servicio de Radiología Oral y Maxilofacial de la de la Clínica Dental Docente de la Universidad Peruana Cayetano Heredia, para obtener los permisos necesarios para acceder a la base de datos de las radiografías, durante el periodo 2015 2017.

Luego el examinador procedió a observar las radiografías y determinó cuáles cumplían con los criterios de inclusión del estudio, discriminando aquellas que no se usarían en la investigación.

Para la observación de las RLED se utilizó una pantalla de 20 pulgadas de la marca Lenovo® y se contó con un ambiente tranquilo y semioscuro. Además, se utilizó el programa SIDEXIS XG para analizar las RLED para el correcto diagnóstico, se usó herramientas como zoom, brillo y contraste.

Habiéndose seleccionado las RLED, se utilizó el programa SIDEXIS XG y se maximizaron las imágenes evaluando la $\mathrm{C} 1$ y en caso tenían mineralización total o parcial del PP se las consideró para el grupo de estudio. Luego de ello se accedió a la herramienta "medir ángulos" a través de la barra de menú análisis. Se seleccionó el primer punto cefalométrico del ángulo $\mathrm{ANB}$, es decir el punto $\mathrm{N}$ y los dos siguientes puntos cefalométricos A y $\mathrm{B}$ determinando el tipo de relación esquelética en números romanos, todos los datos obtenidos se registraron en una hoja de cálculo Excel. 
Se utilizó el programa Estadístico SPSS V.22.0 para Windows. Los resultados estadísticos tuvieron un nivel de significancia de $(\mathrm{P} \leq 0,05)$. La estadística descriptiva de la edad y tipos de PP fue determinada a través de su distribución de frecuencias y porcentajes. La estadística analítica se usó para la comparación de la variable ponticulus posticus en cuanto a presencia según sexo, edad, grupo etario, tipo-sexo y relación esquelética se usó la prueba de Chi Cuadrado.

El presente estudio utilizó información registrada en las bases de datos digitales del Servicio de Radiología Oral y Maxilofacial, de la sede de Salaverry, de la Clínica Dental Docente de la Universidad Peruana Cayetano Heredia, en el periodo 2015 - 2017, para lo cual se obtuvo la aprobación del Comité Institucional de Ética de la Universidad
Peruana Cayetano Heredia. Al tratarse de una base de datos se mantuvo en anonimato a los propietarios de las RLED.

\section{RESULTADOS}

Se revisaron 925 RLED del Servicio de Radiología Bucal y Maxilofacial, de los cuales el 30,6\% (283 casos) presentaron PP y el 69,4\% (642 casos) no lo presentaron, la distribución de la frecuencia del PP según sexo se presenta en la tabla 1.

La distribución de las frecuencias del tipo del PP según sexo y clasificación de Selby et al., (4), se detalla en la tabla 2 y su distribución de frecuencia según edad en la tabla 3 (figura 1)

Tabla 1. Distribución de la frecuencia del ponticulus posticus.

\begin{tabular}{lcccc}
\hline \multirow{2}{*}{ Ponticulus posticus } & \multicolumn{4}{c}{ Sexo } \\
\cline { 2 - 5 } & $\mathbf{n}$ & Masculino & N & Femenino \\
& 226 & $61.6 \%$ & 416 & Frecuencia \\
\hline Ausente & 141 & $38.4 \%$ & 142 & $25.6 \%$ \\
Presente & 367 & $100.0 \%$ & 558 & $100.0 \%$ \\
Total & & &
\end{tabular}

Tabla 2. Distribución de la frecuencia del tipo de ponticulus posticus según sexo.

\begin{tabular}{lcccc}
\hline \multirow{2}{*}{ Tipo de } & \multicolumn{3}{c}{ Sexo } \\
\cline { 2 - 5 } \multicolumn{1}{c}{ Ponticulus posticus* } & \multicolumn{2}{c}{ Masculino } & \multicolumn{2}{c}{ Femenino } \\
\hline Ausente & $\mathbf{n}$ & Frecuencia & 416 & Frecuencia \\
Completa & 226 & $24.4 \%$ & 50 & $45.0 \%$ \\
Parcial & 75 & $8.1 \%$ & 92 & $5.4 \%$ \\
Total & 66 & $7.1 \%$ & 558 & $9.9 \%$ \\
\hline
\end{tabular}

* Clasificación Selby et al. ${ }^{8}$

Tabla 3. Distribución de la frecuencia de tipo de ponticulus posticus según edad.

\begin{tabular}{lcccccc}
\hline \multirow{2}{*}{ Grupo etario } & \multicolumn{5}{c}{ Ponticulus posticus } \\
\cline { 2 - 7 } & $\mathbf{n}$ & $\begin{array}{c}\text { Ausente } \\
\text { Frecuencia }\end{array}$ & $\begin{array}{c}\text { Completa } \\
\text { Frecuencia }\end{array}$ & Parcial & Frecuencia \\
\hline 0 a 9 años & 16 & $1.7 \%$ & 5 & $0.5 \%$ & 8 & $0.9 \%$ \\
10a 19 años & 326 & $35.2 \%$ & 65 & $7.0 \%$ & 84 & $9.1 \%$ \\
20 a 29 años & 179 & $19.4 \%$ & 35 & $3.8 \%$ & 34 & $3.7 \%$ \\
30 a 39 años & 71 & $7.7 \%$ & 15 & $1.6 \%$ & 20 & $2.2 \%$ \\
40 a 49 años & 34 & $3.7 \%$ & 4 & $0.4 \%$ & 7 & $0.8 \%$ \\
50 a 59 años & 13 & $1.4 \%$ & 0 & $0.0 \%$ & 1 & $0.1 \%$ \\
60 a 69 años & 3 & $0.3 \%$ & 1 & $0.1 \%$ & 4 & $0.4 \%$ \\
Total & 642 & $69.4 \%$ & 125 & $13.5 \%$ & 158 & $17.1 \%$ \\
\hline
\end{tabular}




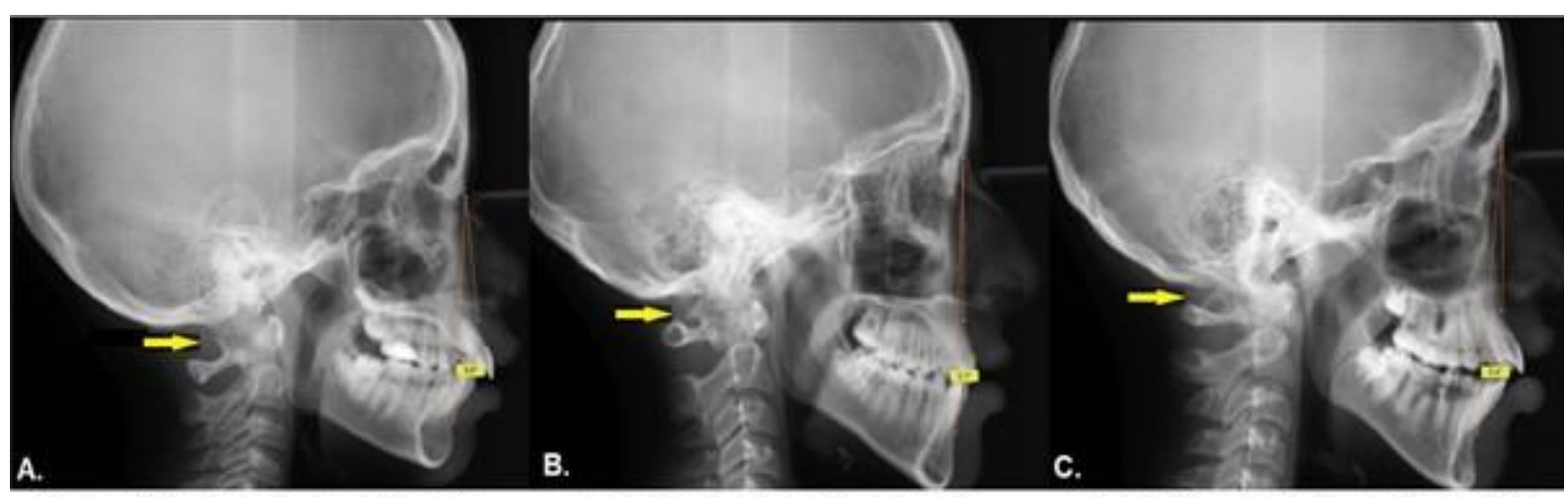

A. Ponticulus Posticus Ausente

B. Ponticulus Posticus Parcial

C.Ponticulus Posticus Completa

Figura. 1. Tipo de ponticulus posticus

Tabla 4. Distribución de la frecuencia del ponticulus posticus según la relación esquelética

\begin{tabular}{lcccccc}
\hline & \multicolumn{3}{c}{ Redacción esquelética } \\
\cline { 2 - 7 } $\begin{array}{l}\text { Ponticulus } \\
\text { Posticus }\end{array}$ & \multicolumn{3}{c}{ Clase I } & & Clase II & \multicolumn{2}{c}{ Clase III } \\
& 212 & \% del total & $\mathbf{n}$ & \% del total & n & \% del total \\
\hline Ausente & 92.9 & 402 & 43.5 & 28 & 3.0 \\
Presente & 308 & 10.4 & 177 & 19.1 & 10 & 1.1 \\
Total & 33.3 & 579 & 62.6 & 38 & 4.1 \\
\hline
\end{tabular}

Tabla 5. Estudios recientes de la frecuencia del ponticulus posticus con diferentes técnicas.

\begin{tabular}{ccccccc}
\hline Autores & Año & País & $\begin{array}{c}\text { Total de pacientes } \\
\text { examinados }\end{array}$ & $\begin{array}{c}\mathbf{N}^{\circ} \text { de pacientes } \\
\text { con PP }\end{array}$ & $\%$ & $\begin{array}{c}\text { Técnica } \\
\text { Imagenológica }\end{array}$ \\
\hline Cook & 2018 & Perú & 925 & 283 & 30.6 & RLED \\
Vanek et al. & 2017 & Czech & 511 & 73 & 14.3 & TCA \\
Adisen et al. & 2016 & Turquía & 1246 & 234 & 18.8 & RL \\
Chen et al. & 2015 & Taiwan & 500 & 35 & 7 & TCHC \\
Ercan et al. & 2015 & Turquía & 698 & 257 & 36.8 & TCHC \\
Elliot et al. & 2014 & EEUU & 21789 & 3639 & 16.7 & TC-RL \\
Mudit et al. & 2014 & India & 650 & 72 & 11.1 & RL \\
Pérez et al. & 2014 & Perú & 1056 & 209 & 19.8 & RL \\
Bayrakdar et al & 2014 & Turquía & 730 & 127 & 17.4 & TCHC \\
Elgafy et al. & 2014 & EEUU & 100 & 38 & 38 & TC \\
Chitroda et al. & 2013 & India & 500 & 342 & 68.4 & RL \\
Schilling et al. & 2010 & Chile & 436 & 84 & 19.3 & RL \\
Wight et al. & 1999 & UK & 895 & 161 & 18 & RL \\
\hline
\end{tabular}

PP: Ponticulus posticus.

TCHC: Tomografía computarizada de haz cónico

RL: Radiografía lateral.

TCA: Tomografía computarizada angiográfica.

RLED: Radiografía lateral estricta digital. 
Tabla 6. Estudios recientes de la frecuencia del ponticulus posticus (presente y ausente) según sexo.

\begin{tabular}{|c|c|c|c|c|c|c|c|c|c|c|}
\hline \multirow{3}{*}{ Autores } & \multirow{3}{*}{ Año } & \multirow{3}{*}{ País } & \multicolumn{8}{|c|}{ Sexo } \\
\hline & & & \multicolumn{4}{|c|}{ PP Masculino } & \multicolumn{4}{|c|}{ PP Femenino } \\
\hline & & & Pte. & $\%$ & Ausente & $\%$ & Pte. & $\%$ & Ausente & $\%$ \\
\hline Cook & 2018 & Perú & 141 & 38.4 & 226 & 61.6 & 142 & 25.4 & 416 & 74.6 \\
\hline Chen et al. ${ }^{5}$ & 2015 & Taiwan & 12 & 5.1 & 235 & 88 & 23 & 9.5 & 219 & 90.5 \\
\hline Ercan et al. ${ }^{16}$ & 2015 & Korea & 129 & 41.2 & 184 & 58.8 & 128 & 33.2 & 257 & 66.8 \\
\hline Pérez et al. ${ }^{6}$ & 2014 & Perú & 101 & 22.1 & 356 & 77.9 & 108 & 19.3 & 451 & 80.7 \\
\hline Bayrakdar et al. ${ }^{3}$ & 2014 & Turquía & 54 & 19.5 & 223 & 80.5 & 73 & 16.1 & 380 & 83.9 \\
\hline Chitroda et al. ${ }^{13}$ & 2013 & India & 154 & 58 & 112 & 42 & 148 & 63.2 & 86 & 36.8 \\
\hline Schilling et al. ${ }^{10}$ & 2010 & Chile & 28 & 10.6 & 235 & 89.4 & 15 & 8.7 & 158 & 91.3 \\
\hline Selby et al. ${ }^{8}$ & 1955 & EEUU & 89 & 69.5 & 39 & 30.5 & 134 & 75.2 & 44 & 24.5 \\
\hline
\end{tabular}

PP: Ponticulus posticus.

Pte. : Presente

Porcentajes hallados con los pacientes que presentaban ponticulus posticus.

Tabla 7. Resumen de la frecuencia del ponticulus posticus (presente y ausente) según grupos etáreos.

\begin{tabular}{|c|c|c|c|c|c|}
\hline \multirow[b]{2}{*}{ Autores } & \multirow[b]{2}{*}{ Año } & \multirow[b]{2}{*}{ País } & \multicolumn{3}{|c|}{ Ponticulus posticus } \\
\hline & & & Grupo etáreo & Presente\% & Ausente\% \\
\hline \multirow{6}{*}{ Cook } & \multirow{6}{*}{2018} & \multirow{6}{*}{ Perú } & $0-9$ & 1.4 & 1.7 \\
\hline & & & $10-19$ & 16.1 & 35.2 \\
\hline & & & $20-29$ & 7.5 & 19.4 \\
\hline & & & $30-39$ & 3.8 & 7.7 \\
\hline & & & $40-49$ & 1.2 & 3.7 \\
\hline & & & $50-59$ & 0.1 & 1.4 \\
\hline \multirow{8}{*}{ Chen et al. ${ }^{5}$} & \multirow{8}{*}{2015} & \multirow{8}{*}{ Taiwan } & $\geq 80$ & 0.5 & 0.3 \\
\hline & & & $20-29$ & 22.8 & 12.3 \\
\hline & & & $30-39$ & 5.7 & 10.5 \\
\hline & & & $40-49$ & 25.7 & 21.9 \\
\hline & & & $50-59$ & 37.1 & 29.9 \\
\hline & & & $\geq 60$ & 8.5 & 25.4 \\
\hline & & & $5-18$ & 13 & 87 \\
\hline & & & $\geq 19$ & 24.8 & 75.2 \\
\hline \multirow{2}{*}{ Pérez et al. ${ }^{6}$} & \multirow{2}{*}{2014} & \multirow{2}{*}{ Perú } & $8-18$ & 14.1 & 85.9 \\
\hline & & & $19-28$ & 17.7 & 82.3 \\
\hline \multirow{2}{*}{ Bayrakdar et al. ${ }^{3}$} & \multirow{2}{*}{2014} & \multirow{2}{*}{ Turquía } & $29-38$ & 17.3 & 82.7 \\
\hline & & & $39-48$ & 19.6 & 80.4 \\
\hline \multirow{8}{*}{ Schilling et al. ${ }^{10}$} & \multirow{8}{*}{2010} & \multirow{8}{*}{ Chile } & $49-81$ & 21.7 & 78.3 \\
\hline & & & $0-10$ & 86.9 & 13.1 \\
\hline & & & $11-20$ & 52.8 & 47.2 \\
\hline & & & $21-30$ & 80.1 & 19.9 \\
\hline & & & $31-40$ & 93.8 & 6.2 \\
\hline & & & $41-50$ & 98.4 & 1.6 \\
\hline & & & $51-60$ & 99.5 & 0.5 \\
\hline & & & $61-70$ & 99.8 & 0.2 \\
\hline
\end{tabular}

PP: Ponticulus posticus.

Porcentajes hallados con los pacientes que presentaban ponticulus posticus. 
Tabla 8. Resumen de la frecuencia del tipo ponticulus posticus según sexo.

\begin{tabular}{|c|c|c|c|c|c|c|c|c|c|c|}
\hline \multirow{3}{*}{ Autores } & \multirow{3}{*}{ Año } & \multirow{3}{*}{ País } & \multicolumn{8}{|c|}{ Sexo } \\
\hline & & & \multicolumn{4}{|c|}{ PP Masculino } & \multicolumn{4}{|c|}{ PP Masculino } \\
\hline & & & Completo & $\%$ & Parcial & $\%$ & Completo & $\%$ & Parcial & $\%$ \\
\hline Cook & 2018 & Perú & 75 & 8.1 & 66 & 7.1 & 50 & 5.4 & 92 & 9.9 \\
\hline Gibelli . ${ }^{18}$ & 2015 & Italia & 8 & 8.8 & 10 & 11 & 9 & 6.9 & 10 & 7.7 \\
\hline Ercan et al. ${ }^{16}$ & 2015 & Turquía & 26 & 8.3 & 52 & 16.6 & 18 & 4.7 & 61 & 15.8 \\
\hline Ercan et al. ${ }^{4}$ & 2015 & Turquía & 21 & 8.5 & 43 & 17.4 & 15 & 5.1 & 57 & 19.3 \\
\hline Mudit et al. ${ }^{17}$ & 2014 & India & 8 & 2.8 & 22 & 7.6 & 11 & 3.1 & 31 & 8.6 \\
\hline Pérez et al. ${ }^{4}$ & 2014 & Perú & 53 & 5 & 55 & 5.2 & 39 & 3.7 & 62 & 5.9 \\
\hline
\end{tabular}

PP: Ponticulus posticus.

Porcentajes hallados con los pacientes que presentaban ponticulus posticus.

Con respecto a las frecuencias del PP y la relación esquelética tenemos que la mayor fue en la clase II con 43,5\% (402 casos), seguido de la clase I con $22,9 \%$ (212 casos) y la clase III con 3,0\% (28 casos), y en relación al PP presente y la relación esquelética tenemos que el de mayor frecuencia fue en la clase II con $19,1 \%$ (177 casos), seguido de la clase I con $10,4 \%$ (96 casos) y la clase III con $1,1 \%$ (10 casos). No existió diferencia significativa entre los grupos de relación esquelética (tabla 4).

\section{DISCUSIÓN}

El presente estudio es uno de los pocos a nivel mundial y el primero en el país en el cual se evalúan además de la presencia del PP, su asociación con la relación esquelética tratando de buscar posibles relaciones con las maloclusiones.

La frecuencia del PP ha sido reportada por diferentes investigadores en países del mundousando variadas técnicas imagenológicas, con una variación general del $7 \%$ a $68.4 \%$ en Taiwán y la India. Sin embargo, los rangos de frecuencia del PP entre $11,1 \%$ y $19 \%$ se presentan en varios trabajos de investigación $(3,5,6,8,9,10,11,12,13,14,15)$. En el presente estudio en las RLED se encontró una frecuencia del PP de $30.6 \%$ este resultado fue superior a los valores encontrados en estudios realizados en Chile, Turquía y Reino Unido, sin embargo, en la India se presentó en el $68,4 \%$ de los casos $(6,13,15)$. Se podría explicar estos resultados por variaciones étnicas o genéticas, también por des uniformidad del tamaño de las muestras estudiadas.

En cuanto al sexo los estudios mundiales muestran diferentes valores de frecuencia del PP, autores como
Chen et al., Chitroda et al. y Selby et al. quienes indican que el PP se presentó con mayor frecuencia en las mujeres $(4,6,13)$, pero en las investigaciones de Ercan, Pérez, Bayrakdar y Schiling se encontró que existió un mayor predominio del PP en los varones, que coincide con la presente investigación con porcentajes de $38,4 \%$ y $25,1 \%$ del PP tanto para varones como mujeres $(3,5,7,10)$ (tabla 6$)$, ningún autor consultado en la bibliografía hace referencia a la causa de este hallazgo.

Pérez et al. encontraron para los rangos etéreos de 5-18 años $13 \%$ y $^{3} 19$ años 24,8\% de PP presente (7). En este estudio no se realizaron estudios de la relación esquelética en dicho rango de edad, sin embargo se encontraron resultados parecidos en los diferentes tipos de PP, así como también en la presencia o no del PP, cabe resaltar que la muestra del estudio de Pérez et al., fue de 1056 (7), y del presente fue de 925 RLED.

Para Chen et al.,sus resultados en grupos etáreos entre 20 a 60 donde prevalecieron los grupos de 50 a 59 años con $37.1 \%$ y de 40 a 49 con $25.7 \%$ de presencia de PP (6).

Bayrakdar et al., encontraron que las variaciones en la frecuencia para PP presente fueron de $14,1 \%$ a $21,7 \%$ para las edades comprendidas entre 8 a 81 años (3).

Para la edad la presencia del PP ha sido reportada en diferentes grupos etéreos por Schilling et al., entre 10 y 70 años encontrándose valores de PP presente de $52 \%$ a $99.8 \%$ y ausente de $0.2 \%$ a $47 \%$ en las edades de 11 a 70 años (10). 
En el presente estudio se observó también que la distribución de la frecuencia de PP en grupos etáreos de 10 a 69 años fue mayor en el grupo de 10 a 19 años con $51,4 \%$ y de 21 a 30 años con $26.8 \%$, parecidos a los encontrados por Bayrakdar et al., (3). En cuanto al tipo de PP encontrados para el sexo masculino el porcentaje de frecuencia del PP completo oscilo entre $5 \%$ a $8,8 \%$ mientras para la formación parcial fue de 5,2 a $17,4 \%$, para las mujeres fueron: PP completo de $3,1 \%$ a $6,9 \%$ y para PP parcial de $5,9 \%$ a $19,3 \%$ respectivamente.

El tipo de PP según el grupo etáreo en el presente estudio mostró que el rango de 10 a 19 años tuvo $7 \%$ de PP completo y parcial $9.1 \%$ presentándose el de tipo completo en los del sexo masculino y parcial en los de sexo femenino que coinciden con los estudios realizados por Gibelli, Ercan et al. y Pérez $(7,16,17)$, pero diferentes a los encontrados en la investigación de Mudit et al. donde los varones tuvieron una mayor frecuencia de PP tipo parcial (15).

En cuanto al PP y la relación esquelética Gutiérrez et al., (18), muestra 680 radiografías laterales de cráneo de la Especialidad de Ortodoncia de la Universidad Autónoma de Nayarit tomadas del 2010 al 2015 donde encontraron que en la población clase I el 8,3\% presentó PP, en la población clase III el $13.04 \%$ presentó PP y en la población clase II fue la de mayor porcentaje con $36,2 \%$; valores similares a los encontrados en nuestra investigación la cual muestra el mayor porcentaje de PP presente con la maloclusión clase II con 19,1\%.

Las variaciones encontradas al comparar en cada uno de los objetivos los valores de la frecuencia del $\mathrm{PP}$ con los reportados por lo diferentes investigadores mencionados nos indican que se deban posiblemente al tamaño de la muestra, la diferencia de edades muestreadas, al tipo de vida diaria (alimentación, enfermedades de los pacientes, etc.) y a los rasgos étnicos, genéticos de la población citadina de origen heterogéneo que tuvimos en el presente estudio.

Se puede apreciar actualmente que es difícil establecer una pauta, tener un orden de relaciones de los diferentes trabajos de investigación sobre el PP, en diferentes países, varía el interés del estudio y las variables analizadas y realizadas, sin embargo a partir del presente trabajo se podría proponer para en un futuro, estimar, por ejemplo una población peruana en forma ordenada por edad, sexo, origen (nativos o mestizos) con el fin de poder ir estableciendo criterios de uniformización que permitan profundizar sobre el PP.

\section{CONCLUSIONES}

El PP es una variante anatómica que se presentó en el 30,6\% de los casos. No se encontró diferencias significativas entre los grupos etáreos ni relación esquelética. En cuanto al sexo no se encontró diferencias estadísticas significativas respecto al tipo de PP pero si en cuanto a la presencia y ausencia de PP siendo mayor en el sexo masculino.

\section{Correspondencia:}

Jonathan Cook García Blásquez

Las Violetas 120, Urb. Jardines Virú, Bellavista, Callao.

Correo Electrónico: jona81017@ @otmail.com

\section{REFERENCIAS BIBLIOGRAFICAS}

1. Capelozza L. Diagnóstico en ortodoncia. Maringá: Dental Press; 2005.

2. Testut L, Laterjet, A. Compendio de anatomía descriptiva. Barcelona: Salvat; 2013.

3. Bayrakdar I, Miloglu O, Altun O, Gumussoy I, Durna D, Yilmaz A. Cone beam computed tomography imaging of ponticulus posticus: prevalence, characteristics, and a review of the literature. Orar Surg Oral Med Oral Pthd Radiol. 2014;118:210-219.

4. Selby S, Stanley MG, Kanareft V. The incedence and familian, nature of a bony bridge on the first cervical vertebra. Am J Phys Anthropol. 1955;49:193-203.

5. Ercan A, Soylu E, Payveran M, Sami O. Is there a relationship between the presence of ponticulus posticus and elongated styloid process? Clin Imaging. 2015; 39(2):220-4.

6. Chen $\mathrm{CH}$, Chen YK, Wang CK. Prevalence of ponticuli posticus among patients referred for dental examinations by cone-beam CT. Spine J. 2015;15(6):1270-6.

7. Pérez I, Chávez A, Ponce D. Frequency of ponticulus posticus in lateral cephalometric radiography of Peruvian Patients. Int J Morphol. 2014;32(1):54-60.

8. Vanek P, Bradác O, De-Lacy P, Konopkova R, Lacman $\mathrm{J}$, Benes V. Vertebral artery and osseous anomalies characteristics at the craniocervical junction diagnosed by $\mathrm{CT}$ and 3D CT angiography in normal Czech population: analysis of 511 consecutive patients. Neurosurg Rev. 2017; 40: 369.DOI: 10.1007/s10143- 
016-0784-x

9. Wight S, Osborne N, Breen AC. Incedence of ponticulus posterior of the atlas in migraine and cervicogenic headachy. J Manipulative Physiol Ther. 1999; 22(1):1520.

10. Schilling J, Schilling A, Suazo GI. Ponticulus posticus on the posterior arch of atlas, prevalence analysis in asymptomatic patients. Int J Morphol. 2010;28(1):31722.

11. Elliot RE, Teanweer $O$. The prevalence of the ponticulus posticus (arcuate foramen) its importance in the Goel - Harms procedure: meta - analysis and review of the literature. World Neurosurg. 2014; 82(1):335-43

12. Elgafy H, Pompo F, Vela R, Elsamaloty H. Ipsilateral arcuate foramen and high-ridign vertebral artery: Implication on C1 - C2 instrumentation. Spine J. 2014; 14(7):1351-5.

13. Chitroda PK, Katti G, Baba IA, et al. Ponticulus posticus on the posterior arch of atlas, prevalence analysis. J Cli Diagn Res. 2013;7(2):3044-7.

14. Adisen MZ, Misirlioglu M. Prevalence of ponticulus posticus among patients with different dental malocclusions by digital lateral cephalogram: a comparative study. Surg Radiol Anat. 2016;39(3):2937.
15. Mudit G, Srinivas K, Sathessha R. Retrospective analysis of Ponticulus Posticus in Indian orthodontic patients-A lateral cephalometric study. Ethiop J Health Sci. 2014; 24(4): 285-290.

16. Ercan A, Soylu E, Payveren M, Ozcan G, Amuk M, Kocoglu F. Prevalence and Morphologic Characteristics of Ponticulus Posticus: Analysis Using Cone-Beam Computed Tomography. Journal of Chiropractic Medicine. 2015;14:153-61.

17. Gibelli D, Capella A, Cerutti E, Spagnoli L, Dolci C, Sforza C. Prevalence of ponticulus posticus in a Northern Italian orthodontic population: a lateral cephalometric study. Surg Radiol Anat. 2016; 38:309. DOI: 10.1007/s00276-015-1554-0.

18. Gutiérrez M, Gutiérrez J, Gutiérrez J. Ponticulus Posticus en las maloclusiones esquéleticas. Rev Tamé. 2016;5(13):473-6.

Recibido : 15-05-2020

Aceptado : 15-11-2020 